\title{
Evidence-based treatment guidelines: at work in a microcosm
}

Patricia Whelan

Work Loss Data Institute, LLC, Encinitas CA 92024, USA. patriciawhelan@aol.com

Background: Medical treatment under workers' compensation represents just a miniscule portion (about 1 percent) of total medical costs in the U.S. Furthermore, legislation and rules are determined autonomously by each state.

Objective: To study Workers' Comp systems in each state and determine what, if any, impact states adopting the use of evidence-based treatment guidelines has had to outcomes within the respective states. Hypothesis: Worker' comp medical care, and the outcomes of that care in each state, can theoretically represent a microcosm of what could be achieved in an entire country.

Methods: A trend began in 2003, starting with California, for states to consider adopting Evidence-Based Treatment Guidelines EBTGs) as a mechanism to insure timely and quality care for injured workers by following the least invasive, most-effective treatments today's science has to offer. Bi-products to the effective implementation of EBTGs, include earlier return to work, better outcomes resulting in reduced indemnity costs, less friction in the system (providers know what treatments are authorized and will be paid for), fewer episodes of over-utilization of services, and decreased medical costs, benefiting employers, insurers, providers and business. Findings: Outcomes where true EBTG have been implemented: Ohio adopted EBTGs in 2003. A Pilot conducted in 2005 showed a decrease in medical costs by $64 \%$, lost days by $69 \%$ and treatment delays by $77 \%$. Texas adopted EBTG in 2006 ; Total costs have declined by $50 \%$, patients are recovering more quickly, more providers are willing to treat these patients, opioid abuses have declined and many states are looking to adopt this model.

Discussion: Other state outcomes and essential elements needed in an EBTG will be discussed, time permitting.

Conclusion: Outcomes realized through the adoption/implementation of evidence-based treatment guidelines by selected states, need not be isolated to the US or to the "microcosm" of workplace injuries and illnesses. Countries adopting evidence-based treatments guidelines within their healthcare systems can benefit from the same results: more timely and quality care, better outcomes, reduced costs, less disputes, reduced utilization of unnecessary procedures and services. 